\title{
Perspectivas de Terapia Ocupacional frente al VIH/SIDA: Una Experiencia de VIHDA
}

Aida del Pilăr Becerrà Becerra' Maribel Moreno Sosa²

Este escrito presenta 10 s a portes generados a par. tir de la pasantiassistematizada de corte expló ratorio cualitattvo, dinguda a fombres y mujeres

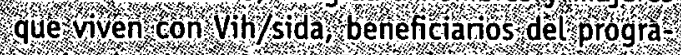
ma institucional Seguro social glinica San pedro Gaver se describe la expenencia de diseno, eje cución y sistematización del programa/y pasantia VIHDA, a paitir de lo cualls se proponen alterna? tivas de trabajo de terapia ocupacional con esta pobblacion.

Se reconoce como urgente un mayor posicionamiento de Ja profesion frente al Vihis sida , zasi como la recesidad de actuar de manera oportuna y transdisciplinana en favor de esta poblacion en aumento, y en pro de la prevention y control de esta realidad cuyo in pacto trasciendede lo per sonala lo socialy de lo local a lo global.

Se desanrollan cinco puntos: 1 . presentación

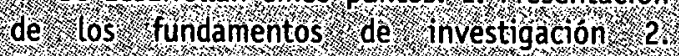
Antecedentes 3 . planteamiento de cpoximaciones

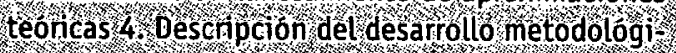
co y 5 . conclusiones y reconnendaciones.
This setter presents the generated contributions from o systematized posantia with qualitative ond explorative onentation, lead to men and women who live with hiv/aids, who are benefia vary of the Seguro Socal and clinical San Pedro. Qlaver institutional program. We desch be the expenence on the design execution and systemati. Zation of the program / pasantia VIIHDA, from which we purpose alternatives of work for the occupational therapy on this population.

Its recognized as urgent a more professional position before the HIV/AIDS, together with the need to acting opportune and interdiscolin ary way ing favour of this growing population. as also. or the prevention and control of the conaition the world, wich have wide effect from both personal to social, and from local to globall situations.

We develop five points. 1 foundations of re search. 2. Antecendent, 3 planning some theo retical approximations, 4. Description of the methodological development ond 5 . Conclisions and recommendations 


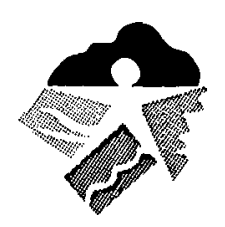

"Cada forma de vida está marcada no sólo por el ajuste al medio ambiente, sino por la rebelión contra ese ambiente: es a la pez criatura y creador; a la vez pictima de la fortuna y ducño del destino."

Patrick Geddes

\section{1•Presentación de los fundamentos de la Investigación}

Veintiún años después de la notificación de la primera prueba clínica del Síndrome de inmunodeficiencia adquirida (Sida), más de 60 millones de personas se han infectado por el Vih y el Sida ha pasado a ser la cuarta causa de mortalidad en el mundo (1). Se estima que para el final del 2003 en todo el planeta habrá 46 millones de personas viviendo con Vih. En Colombia se encuentran 40.072 casos reportados de personas viven con esta condición de salud. (2)

Este panorama y los datos en aumento reportados por el Programa Conjunto de las Naciones Unidas sobre el VIH/SIDA (ONUSIDA) permiten deducir el avance vertiginoso y el fuerte impacto sobre la sociedad contemporánea. Se ha identificado la propagación del Vih/sida como un "peligro" cuyas consecuencias inciden sobre el bienestar, progreso y estabilidad social y económica de las naciones. La consulta a especialistas, frecuentes hospitalizaciones, medicamentos de alto costo, tratamientos prolongados, toma de exámenes especializados y pensiones por invalidez, son algunos de los procedimiento de alto costo requeridos por estas personas.

Pero las implicaciones van más allá del manejo de lo biológico o individual que en fases crónicas o agudas se pueda hacer. Se permean

\section{PALABRAS CLAVE}

diversas dimensiones a partir de las cuales se establecen nuevas demandas/expresiones a nivel afectivo, laboral, escolar y recreativo, requiriendo establecer redes de apoyo familiar, social e institucional.

Es ante estas condiciones, que se propone vislumbrar el vivir con Vih/Sida desde una perspectiva humanista, participativa e inclusiva, con la cual se trasciendan las actitudes que se oponen a la realización humana, como lo son reacciones de temor al otro por ser identificado como portador, el señalamiento profesional/ familiar, la negación de si mismos y de la condición de salud, la perdida de sentido de vida.

La conjunción de estas situaciones, hace que el desempeño ocupacional como expresión del quehacer humano, sufra grandes cambios que es importante conocer y comprender, razón por la cual se planteo como eje de investigación el siguiente problema: ¿Cuáles son los aportes y saberes teóricos y prácticos que se generan a partir de la sistematización del programa VIHDA?

Para dar respuesta a este cuestionamiento y frente a la necesidad de ahondar en las im-

\footnotetext{
'Terapeuta Ocupacional, Universidad Nacional de Colombia. Postulante Maestría Discapacidad e indusión Social de la Universidad Nacional de
} Colombia. pilihada@lycos.com

${ }^{2}$ Terapeuta Ocupacional, Universidad Nacional de Colombia. CIDIE. demi68@hotmail.com

Vih/sida, enfermedad crónica, psicosocial, desempeño ocupacional 
plicaciones en el desempeño ocupacional de quienes viven con $\mathrm{Vih} /$ sida, se diseño y puso en marcha el programa VIHDA, orientado por los siguientes objetivos:

- Presentar el panorama internacional y nacional en la atención de personas que viven con $\mathrm{VIH} / \mathrm{SIDA}$

- Desarrollar una aproximación teórica en cuanto a las implicaciones individuales y colectivas del VIH/SIDA

- Diseñar un programa de terapia ocupacional para personas que viven con VIH/SIDA

- Diseñar un instrumento para la valoración del Desempeño Ocupacional Realizante.

- Ejecutar un programa de terapia ocupacional para personas que viven con VIH/SIDA.

\section{Panorama internacional \\ y nacional en la atención de personas que viven con $\mathrm{VIH} / \mathrm{SIDA}$}

La participación de terapia ocupacional en programas de atención a personas con VIH/ SIDA ha tenido diferentes visiones, enmarcadas permanentemente en los fundamentos filosóficos de la profesión que sustentan la intervención en la promoción del bienestar $y$ prevención de alteración en el quehacer ocupacional de la persona que recibe su servicio. Partiendo de este supuesto tienen origen diversos programas, en los cuales la ocupación ha sido un medio eficaz para promover una vida más óptima.

Entre ellos se encuentran los realizados por Kent (citado en 3), Murphy (4) y las postulaciones teóricas realizadas por Caicedo (5), Galvis y Veloza (3) y Díaz y cols (6) entre otros. Sin embargo hasta la fecha en Colombia las aproximaciones teóricas o experiencias desde terapia ocupacional con un enfoque humanista en la atención de las personas que viven con VIH/SIDA son mínimas, lo cual pone en evidencia la necesidad y urgencia de incursionar en la atención, producción de conocimiento y elaboración de estrategias que ratifiquen el valor de cada persona como ser individual y a la vez social, único e integral, importante para sí mismo y para quienes le acompañan. Así mismo posicionarse con una con una identidad ética y clara en su quehacer frente a otros miembros de los equipos de salud.

\section{Aproximaciones Teóricas}

Con el fin de proporcionar herramientas teóricas que permitan abordar desde la perspectiva integral la atención a personas que viven con Vih/sida se presentan a continuación los lineamientos orientadores de este trabajo de grado que toman elementos de las ciencias humanas y de una aproximación teórica de terapia ocupacional: el modelo Desempeño Ocupacional Realizante $^{3}$. Con lo anterior se pretende, según lo menciona Trujillo (8), propiciar espacios de discernimiento que pongan a prueba la producción teórica realizada para identificar las competencias que hacen pertinente el actuar de los y las terapeutas ocupacionales frente a esta población.

\subsection{Modelo Desempeño Ocupacional} Realizante ${ }^{4}$ :

El modelo Desempeño Ocupacional Realizante se plantea desde una visión humanistá, en la cual el ser humano esta impulsado por una tendencia hacia la autorrealización, por medio de la ocupación, siendo una construcción individual y progresiva de experiencias de autocuidado, juego y trabajo que la persona aprecia y colma de significado para sí, peró que asi mismo requiere esfuerzo y perseverancia (10). Este modelo da como valor agregado

\footnotetext{
${ }^{3}$ El Modelo de Desempeño Ocupacional Realizante es una propuesta a nivel conceptual (estando relacionado primariamente con las ideas, el pensamiento abstracto y los fundamentos teóricos (7) desarrollado por docentes de la Universidad Nacional de Colombia, para entender el desempeño humano a través de la ocupación.

4 Ultima versión desarrollada por Méndez, Pérez, Trujilloy Zapata. 2001.(9)

${ }^{5}$ En la actualidad, el término humanismo se utiliza para indicar toda tendencia del pensamiento que afirme la centralidad, el valor, la dignidad del ser humano, o que muestre una preocupación o interés primario por la vida y la posición del ser humano en el mundo (6).
}

Revista Ocupación Humana/29 


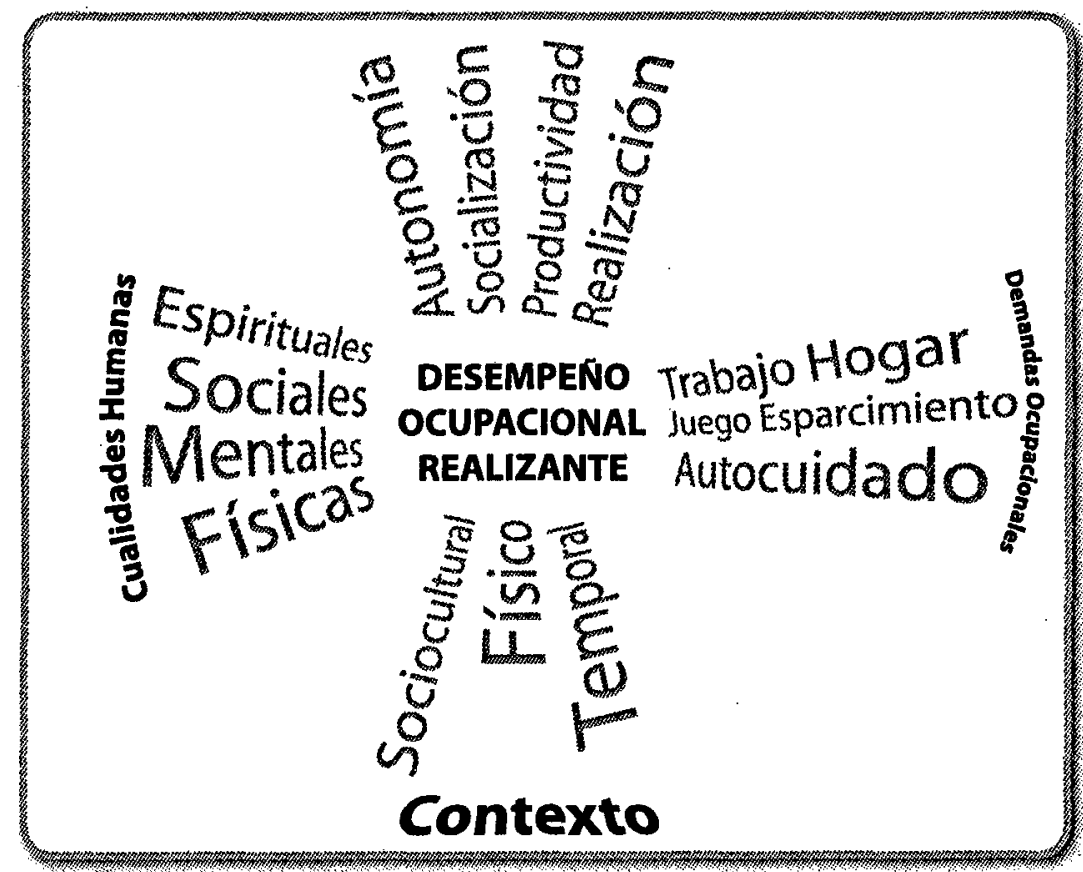

Figura 1. Modelo Desempeño Ocupacional Realizante

Tomado de Grupo Ocupación Humana, Documento en contstrucción. 2001. (9)

la concepción de un valor noético exclusivo al ser humano, complementando así perspectivas anteriormente manejadas en terapia ocupacional.

A partir de la integración dinámica de tres dimensiones (ver figura 1): las cualidades humanas, las demandas ocupacionales y el contexto físico, temporal y sociocultural que intervienen en las experiencias ocupacionales, se construyen los postulados de este modelo.

Con base en estos postulados y de acuerdo con la experiencia en el programa VIHDA se identificaron algunas implicaciones sobre el desempeño ocupacional, ya que el vivir con VIH/SIDA suscita múltiples factores que afectan de forma trasversal las cualidades humanas, el desempeño en las demandas ocupacionales y la promoción en los propósitos de autonomía, socialización, productividad y realización.

En las personas que viven con $\mathrm{VIH} / \mathrm{SIDA}$ la alteración en el desempeño está dado por las condiciones previas de salud ${ }^{6}$, los cambios consecuentes a la infección en las cualidades físicas, mentales, espirituales y sociales, y los generados por el contexto según creencias, costumbres y normas; limitando el grado de satisfacción que producen las ejecuciones y la capacidad de recibir gratificación por personas o situaciones que antes del diagnóstico eran importantes y trascendentales.

La presencia de infecciones oportunistas, el cambio de hábitos necesarios para seguir el tratamiento antiretroviral, la carga emocional al conocer el diagnóstico, el cuestionamiento espiritual al considerar la justicia o injusticia de este, la posible afección a nivel mental al conjugarse factores previos (antecedentes psiquiátricos), actuales (manejo del diagnóstico) y futuros (desarrollo de demencias consecuentes al diagnóstico) y la restricción de sus capacidades sociales al tener que enfrentar el ser portador del virus, coactan la tendencia hacia la realización y afectan no solamente la ejecución satisfactoria de las demandas de automantenimiento, trabajo/estudio/hogar y juego/esparcimiento, sino además valores $y$ conceptos sobre si mismo al sentir que no res-

\footnotetext{
6 Teniendo en cuenta, como lo menciona la OMS, que salud es el bienestar físico, mental y espiritual, y no solamente la ausencia de enfermedad. 'Los comportamientos asumidos pueden manifestarse en la tendencia a replegarse sobre si mismos para eludir la dificultad que supone la interacción con el contexto social en que están inmersos, fomentando el deterioro, ruptura y baja calidad en los vínculos afectivos.
} 
ponde a las exigencias planteadas por el medio, disminuyendo la calidad y sentido de las acciones.

Es necesario aclarar que estas situaciones no solamente son vividas por la persona portadora, sino que se hacen extensivas al interior del grupo social inmediato: quienes conviven con estas personas cumpliendo con roles de padres, pareja, hijos o amigos, compartiendo particularidades y dificultades propias del diagnóstico (deterioro y dependencia física $y$ mental, estigma y rechazo social); y adoptando comportamientos ante los sentimientos de perdida ${ }^{7}$, desconcierto, culpa, miedo, desesperanza, soledad, inutilidad y reproche que se generan al conocer el diagnóstico y contribuyen a agudizar los signos y síntomás de la enfermedad.

Sin embargo el vivir con VIH/SIDA puede brindar una mayor plenitud en el diario vivir cuando la persona ha elaborado sus proceso de duelo y acepta su condición de salud sin culpas ni reproches, se sobrepone a las crisis y supera, en algunos $\operatorname{casos}^{8}$, los pronósticos médicos en cuanto a cantidad de días de vida; con lo anterior se confirman los enunciados de Frankl y del modelo Desempeño Ocupacional Realizante en cuanto a que la tendencia realizante de los seres humanos, según diversas variables, se potencializa o se detiene ante situaciones adversas o límite.

\subsection{Una mirada desde las Ciencias Humanas para el análisis Social}

El VIH/SIDA es una enfermedad que más que destruir físicamente a la persona, altera aspectos no tangibles del ser humano, como son sus afectos, creencias, emociones y deseos. Dicha alteración está directamente relacionada con el origen asignado a la enfermedad, ocasionando la pérdida o disminución en valores

\footnotetext{
' Según FRANKL el ser humano tiene la capacidad de encontrar un significado y un sentido a su existencia en cualquier circunstancia de la vida, aun en los momentos más absurdos y dolorosos (11).

'Desafortunadamente esto no corresponde con el enfoque de las campañas de prevención del VIH/SIDA que se han desarrollado en América Latina, las cuales se han dirigido, en su mayorla a la utilización del condón, más que a la estabilidad de pareja, los valores y afectos, lo cual hace que "sean insuficientes 0 estén en contra de sus objetivos".
}

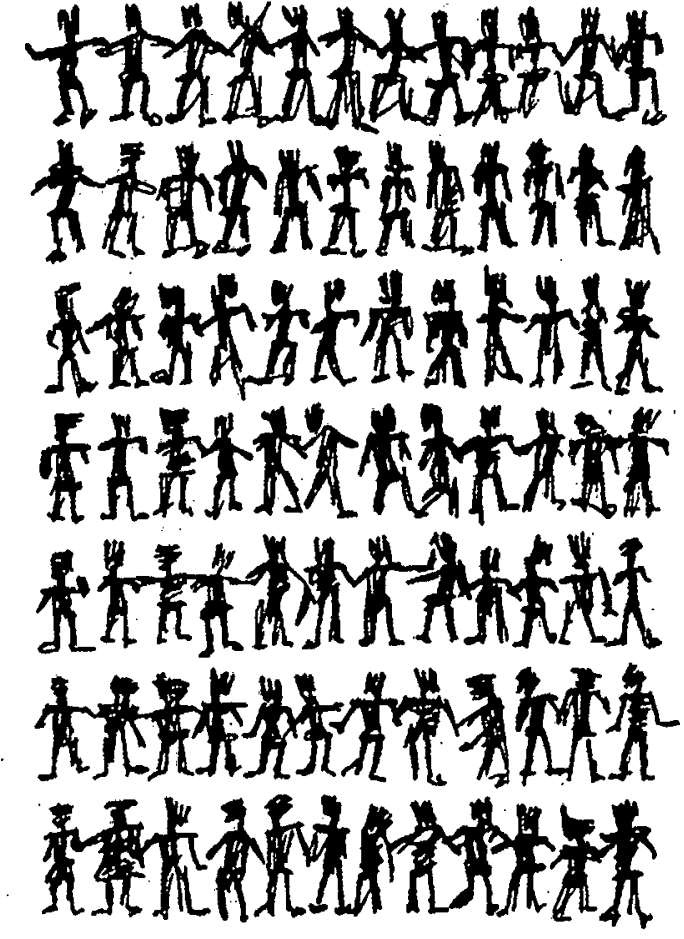

El vivir con VIH/SWA

suscita muiltiples factores que afectan de forma traspersal las cualidades bumanas, el desempeño en las demandas ocupacionales y la promoción cn los propóritos de autonomía, socialización, productividad y realización. 

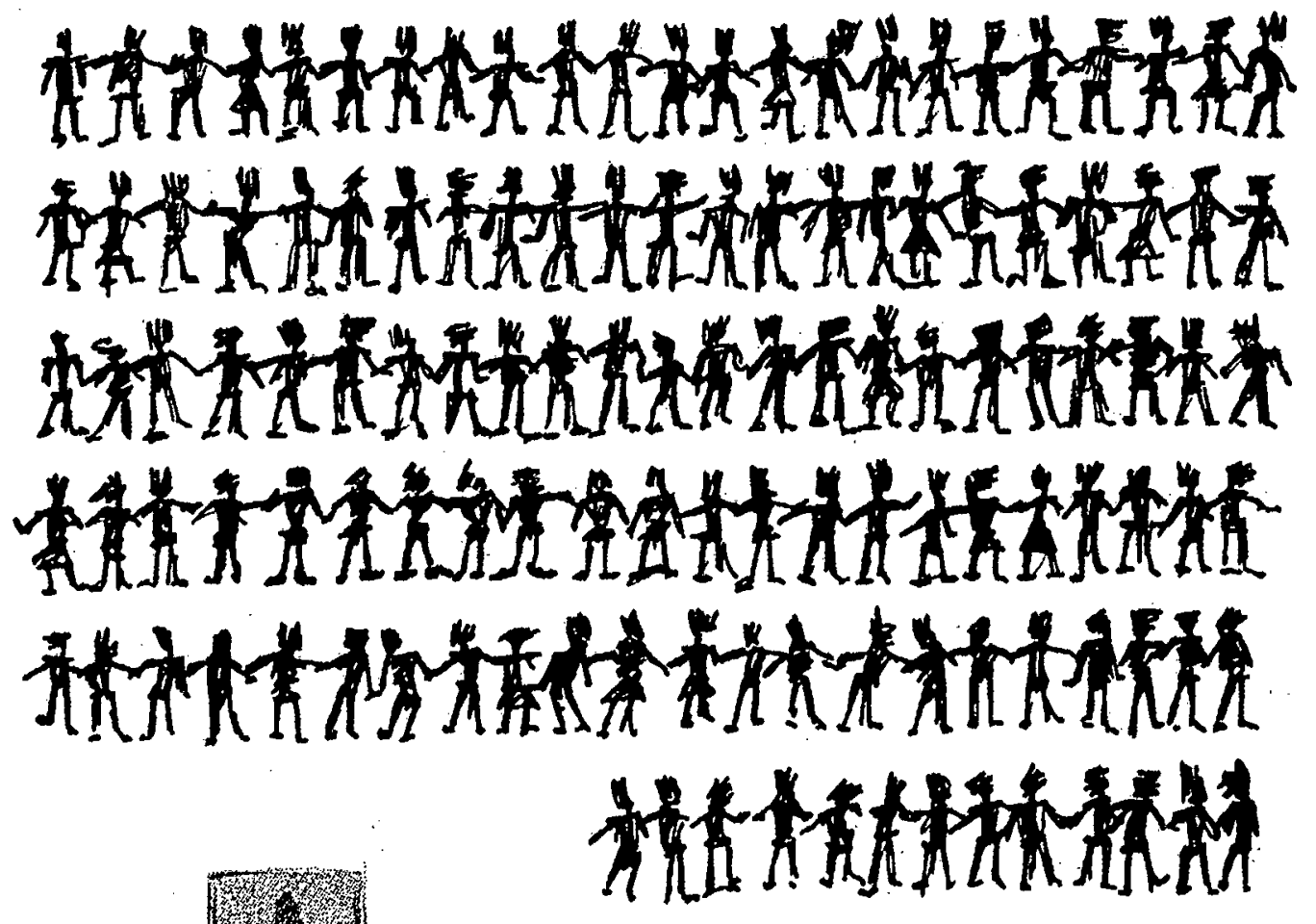

fundamentales para la convivencia; como lo son la confianza, el respeto, la honestidad y el afecto ${ }^{9}$.

Los valores sociales asociados al Vih/sida van ligadas a otras categorías culturalmente cuestionadas: el género (masculino femenino), la orientación sexual (homosexual, bisexual, heterosexual), la clase social, la procedencia, el grupo étnico y el estilo de vida. La conjunción de estas variables permiten entender al Vih/Sida como una "epidemia democrática", que afecta en proporciones tan distintas como distintos son los niños y niñas, hombres y mujeres, que viven con el virus; siendo la ética y el respeto uno de los apoyos más efectivos para la disminución del impacto de la vulnerabilidad generada por el impacto del personal y social que connota esta situación.

La conjunción y presencia de las categorías mencionadas en relación con el VIH/SIDA pueden, en resumen: impedir el acceso a los servicios de salud, propiciar situaciones y conductas de riesgo al contagio como la migración y el desplazamiento, o disminuir la accesibilidad a oportunidades laborales, a programas de información para la promoción de la salud y prevención de la propagación del virus. Lo anterior tiene serias implicaciones en la calidad de vida, en el disfrute en la cotidianidad, en propiciar mayor vulnerabilidad y en afectar la tendencia a la realización de las personas que viven con Vih/Sida.

\section{Desarrollo Metodológico}

Para responder al problema de investigación y desarrollar los objetivos; presentados en la primera parte de este texto, la realización del programa VIHDA, contó con tres momentos metodológicos: la planeación, ejecución y resultados, los cuales se describen en este aparte.

32 Vol.10 No.1 y 22003 


\subsection{Planeación}

A partir del diseño inicial, se presento el proyecto del programa VIHDA a el gerente de clínica San Pedro Claver y la coordinadora del programa de Prevención y Control de Enfermedades de Transmisión Sexual y VIH/ SIDA del Seguro Social, en el cual se desarrollo la pasantía, a partir de las cuales se hicieron ajustes, de tipo metodológico (forma de acceder a la población y distribución horaria)

De acuerdo al objetivo central del programa, promover el desempeño ocupacional realizante en las personas con VIH/SIDA vinculadas programa VIHIDA, se trazaron objetivos específicos acordes con los propósitos ocupacionales y el contexto, como se describe a continuación.

\section{Autonomía}

- Favorecer habilidades para automantenimiento físico, mental, social y espiritual.

- Fomentar desarrollo de actividades de acuerdo a las propias capacidades.

- Brindar estrategias que orienten la toma de decisiones.

\section{Socialización}

- Favorecer participación en actividades de tipo grupal.
- Desarrollar habilidades de comunicación asertiva.

\section{Productividad.}

- Optimizar seńtido de utilidad ên las propias áccionès.

- Orientar en la re-estructuración de rutinas

- Propiciar desarrollo de habilidades productivas.

- Desarrollar habilidades para la utilización significativa del tiempo.

\section{Realización}

- Favorecer búsqueda de sentido de vida.

- Orientar habilidades hacia la satisfacción y:el disfrute de la vida.

\section{Contexto}

- Promover interacción con el contexto familiar para el desarrollo o mantenimiento de sus propósitos realizántes.

- Apoyar al àcudiente en el proceso de aceptación y acompañamiento.

De acuerdo a estos objetivos y a la definición conjunta de criterios de inclusión y exclusión por parte de l@s profesionales del programa del Seguro Social y las pasantes, se inició el proceso de captación de la población. Cuadro 1.

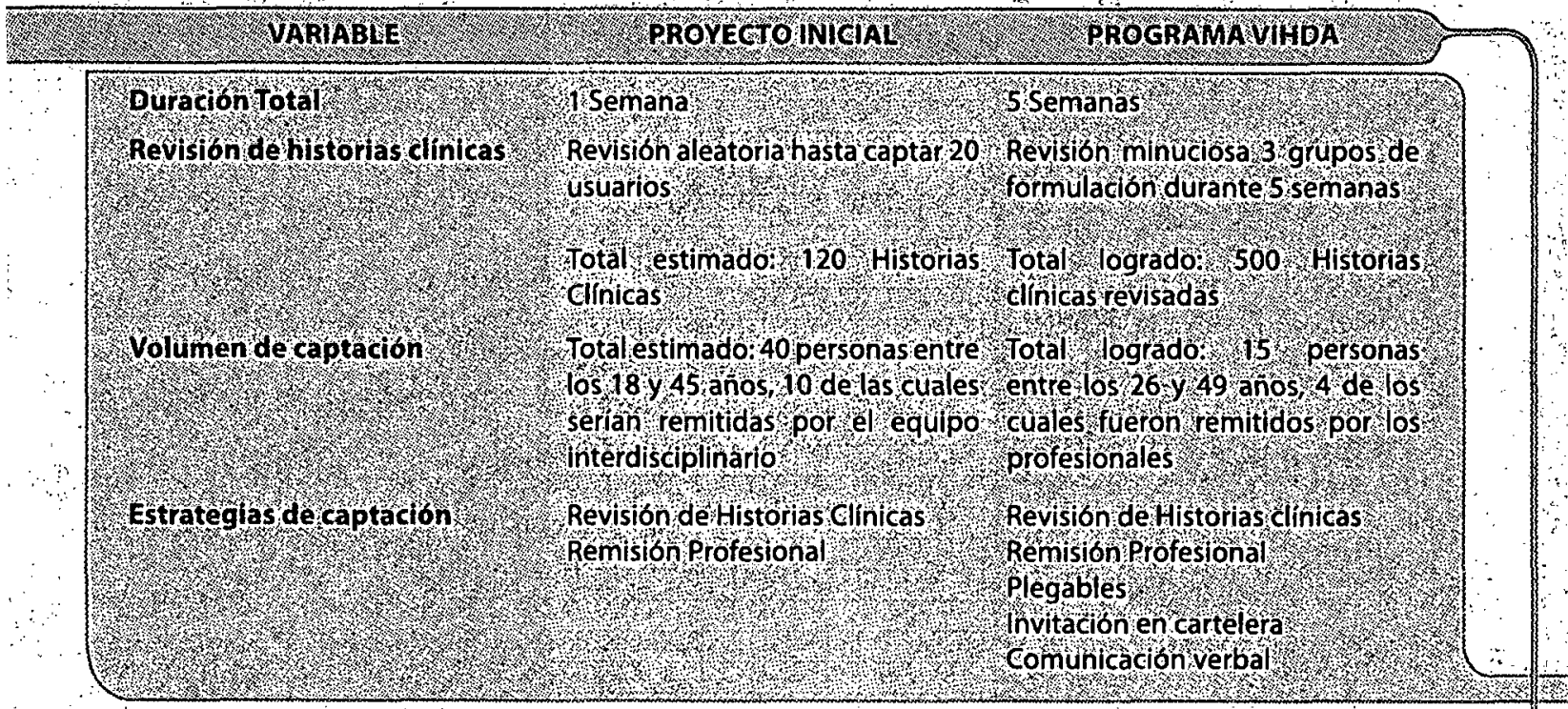

Cuadro 1. Résultados y Adaptaciones en el proceso de captación del prógramá VIHDA 


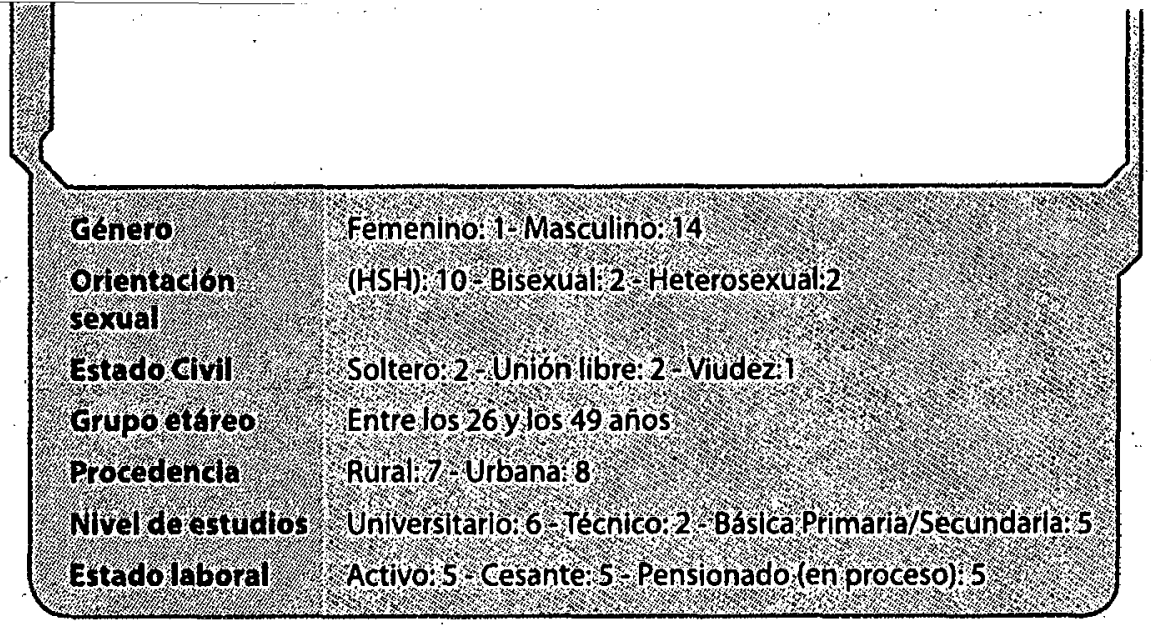

Cuadro 2. Ficha técnica: Grupo participante programa VIHDA

\subsubsection{Valoración de la población}

Una vez finalizado dicho proceso y firmado el consentimiento informado por parte de las personas vinculadas VIHDA, se inició el proceso de la valoración de la población. Ante la necesidad de una estructura evaluativa coherente con los aportes y la población objeto, se diseño, sometió a juicio de expertos y a prueba piloto un instrumento para la valoración del desempeño ocupacional realizante, que incluye un manual, un formato de valoración cualitativa y un formato de valoración cualitativa denominado perfil del desempeño ocupacional, los cuales pueden ser consultados en el trabajo original.

El grupo valorado estuvo conformado por 15 personas, de las cuales presentamos los datos característicos en el Cuadro 2.

Teniendo en cuenta las respuestas dadas en el formato de Valoración del Desempeño Ocupacional y el registro de esta información en el Perfil se identificaron como puntos de intervención prioritaria el propósito de Productividad y la variable de Contexto. Este último fue el de más baja calificación al resaltar el bajo apoyo que los usuarios perciben del contexto a nivel social.

En el propósito de productividad se identificaron como aspectos deficientes que, en la mayoría de los casos, las actividades significativas en las cuales las personas se consideraban buenas no eran realizadas en la actualidad, $y$ que la sensación de desaprovechamiento del tiempo se daba por no tener actividades gratificantes en las cuales ocuparse.

El total de personas que integran el grupo son independientes en las actividades de automantenimiento (cuidado personal, higiene, toma de medicamentos, asistencia médica). Dichas actividades tienen la mayor dedicación por parte del grupo, seguidas en menor proporción por actividades de ocio, el acompañamiento de las personas cercanas y el ejercicio de alguna ocupación remunerada. Se identificaron sentimientos de inconformidad frente a utilización del tiempo, distribución de actividades a lo largo del día, monotonía frente al diario vivir, limitación para la iniciación de nuevas ocupaciones, planteándose como alternativa el entablar nuevas relaciones interpersonales para realizar diversas actividades y disminuir la sensación de soledad. La mayoría de personas activas laboralmente consideraron sus roles laborales actuales no les posibilitan el désarrollo de capacidades ni la aprensión de nuevas habilidades; en el resto, la dificultad para conseguir empleo, la exigencia de certificados de salud por los contratantes, la toma de medicamentos, el temor a enfermar, entre otros, inciden en la no realización de actividades gratificantes y económicamente útiles que les permitían aportar en su contexto familiar $y$ les brinden sentido de utilidad social:

Los propósitos de realización y socialización se presentarón como variables que requieren intervención para su fortalecimiento, al ser un reflejo de las limitantes presentadas en el proceso de productividad $y$ en la variable de contexto. El interactuar y ayudar a otros dan un gran valor a las experiencias de vida de estas personas. El sentirse solos o tristes 
puede se identifica como una causa y también como una consecuencia del aislamiento, la agresividad, le negación y el rechazo tanto de desde y hacia ellos mismos y las personas que les rodean. En la toma de decisiones influyen de manera importante varios aspectos como el dinero, la negación o expresión de su diagnóstico y la opinión y apoyo dado por las personas. La conjugación de estos factores incide en la baja sensación de autonomía de sus actos y se expresa en situaciones percibidas como ajenas del propio control imposibles de manejar.

\subsection{Ejecución del Programa}

Posterior a la Planeación, se puso en marcha el programa VIHDA. Se hicieron los ajustes previstos sobre el proyecto inicial en respuesta a las prioridades de intervención, disponibilidad horaria de los participantes, recursos disponibles y a las necesidades, capacidades, gustos e intereses identificados en la valoración del Desempeño Ocupacional Realizante.

Con el fin de aumentar la cobertura e impacto del programa VIHDA y para implementar acordemente las estrategias de intervención propuestas se enfoco en el desarrollo de atención directa $\mathbf{e}$ indirecta.

\subsubsection{Atención directa}

Se orientó a la facilitación de expresión de capacidades y necesidades, lo cual favoreció el ínicio de un proceso de empoderamiento frente al automantenimiento físico, emocional, espiritual e intelectual al tiempo que interactúa y se confronta con personas que comparten su misma condición de salud. Las acciones desarrolladas fueron las siguientes.

- Acompañamiento Terapéutico Individual: fue un medio de comunicación personalizada y confidencial que permitió establecer una relación terapéutica empática, identificar participativamente estrategias de manejo o prevención de factores de riesgo en el desempeño ocupacional. Para el desarrollo de este acompañamiento se tuvieron en cuenta los aspectos prioritarios identificados en la valoración y las expectativas de la persona, principalmente. Los motivos de consulta, en términos de fueron la pérdida del rol laboral-familiar-de pareja, limitaciones en la distribución del tiempo libre y hábitos y rutinas y bajo autoconcepto, entre otras.
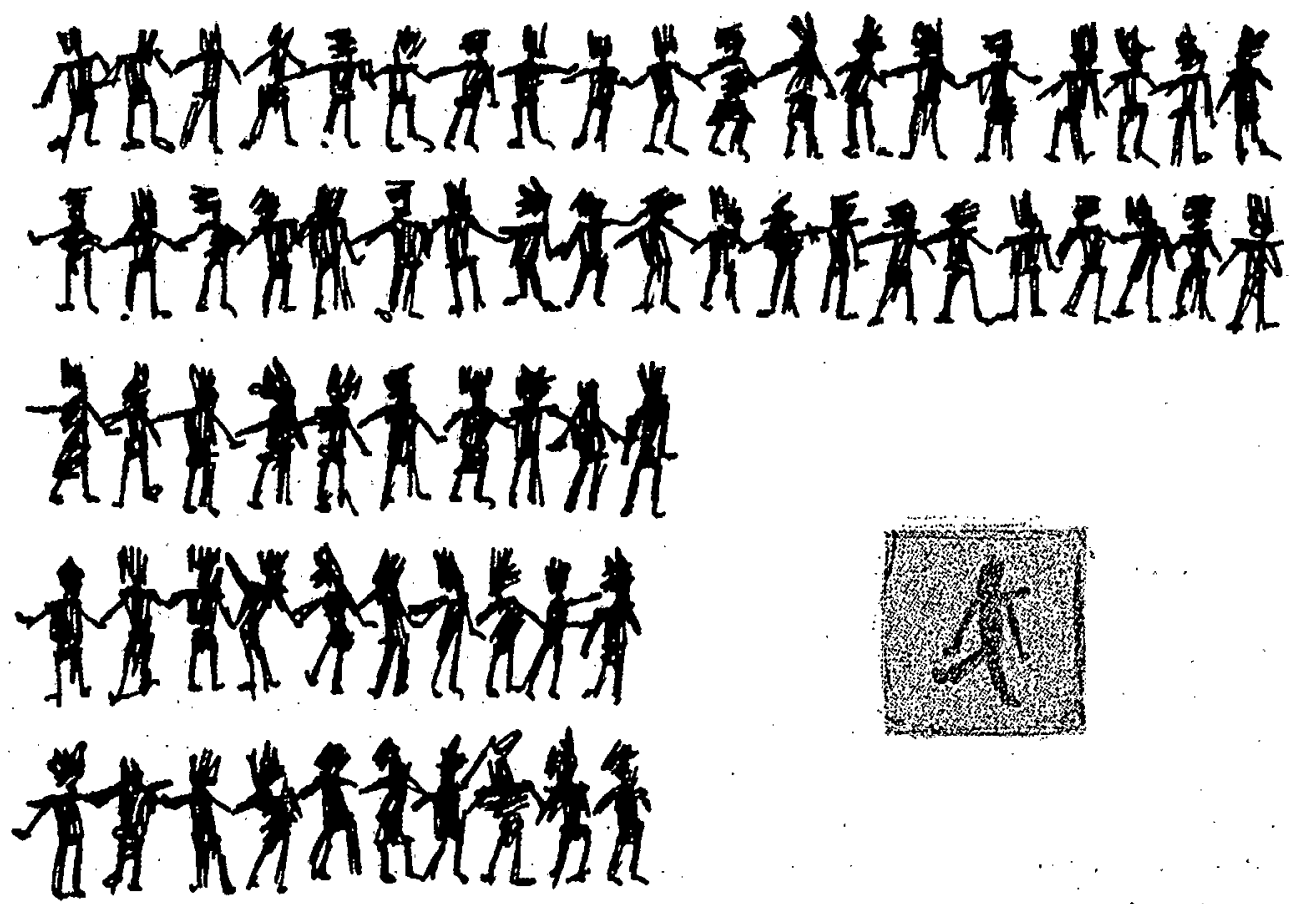

Revista Ocupacion Humana 35 
- Talleres de Socioproductividad (velas y papel artesanal). Se eligieron teniendo en cuenta los riesgos, alternativas y demandas ocupacionales, el manejo terapéutico que permite y el costo de su implementación. Dada la dificultad del programa del Seguro Social para la consecución oportuna de recursos, no fue posible llevar a cabo el taller de Velas Artesanales.

Se llevaron a cabo talleres de elaboración de Elaboración y Productos de Papel (papel artesanal), que permitieron el reconocimiento y desarrollo de habilidades y destrezas, la toma de decisiones colectiva, la participación activa, y el trabajo cooperativo con resultados gratificantes para 1@s participantes. Se orient6 a los participantes en técnicas de ahorro de energía y simplificaciones de trabajo (aplicables y necesarias en los momentos de exacerbación de síntomas) higiene postural y organización del puesto de trabajo. El interés manifiesto por la participación, estuvo dado principalmente por el aprendizaje de una técnica y desarrollo de nuevas habilidades, y el sentido de gratificación y utilidad (económica en algunos casos) otorgado al producto terminado. Se identificó que este tipo de talleres que involucran la actividad con significado y a la vez un producto tangible, tuvieron mayor número de participantes frente a los talleres de autonomía y realización (que se explican a continuación); es posible que esta está dada por la utilidad práctica/ remunerada que representa el producto terminado.

- Talleres de Autonomía y Realización: La temática de estos talleres se estructuró de acuerdo a las prioridades de intervención identificadas en la valoración. Estas actividades permitieron a los participantes confrontar(se), expresar(se), escuchar(se) y retroalimentar(se) a partir de las propias vivencias, compartiendo sus historias, sentires, alegrías y dolores del diario vivir. Las estrategias utilizadas para el desarrollo de estas activi- dades estuvieron orientadas a que en la búsqueda del sentido gratificante en el desempeño ocupacional se incluya como determinantes el (re)conocimiento personal, la autoconciencia corporal, la elaboración de procesos de duelo, la estructuración de hábitos y rutinas saludables, la utilización productiva del tiempo libre y el sentido de vida. Las metodologías y estrategias se apoyaron en la utilización de plegables referentes a los temas a tratar, y que permitían a la vez retroalimentar sobre las experiencias vividas en los talleres.

En el desarrollo de las actividades un factor común fue la baja asistencia, pese a los diversos medios utilizados para contrarrestarla. Presumiblemente una de las causas es que el asistir a grupos terapéuticos está erróneamente relacionado a enfermedad, discapacidad o necesidad de ayuda, lo cual requiere el reconocimiento y aceptación de las diferentes implicaciones del diagnóstico, no siendo evidente en las primeras fases de la enfermedad al encontrarse asintomática. El plantear la necesidad de colaboración y/o ayuda profesional, o el proyectar la discapacidad de otros usuarios en si mismos, confronta a la persona $y$ hace inminente el temor a los cambios funcionales que se pueden generar en el futuro, siendo la no asistencia a la institución o a grupos de apoyo una forma de negación a la situación de salud vivida.

Así mismo, durante el desarrollo de los talleres, fue evidente el temor al cambio o modificación en la estructura de la cotidianidad, que en la mayoría se encontraba bajo rutinas rígidas, al planteamiento de nuevas metas $y$ desarrollo actividades. Dichas actitudes, se justificaban por la disminución en la motivación de vivir, en la confrontación con su propia mortalidad, en el temor al rechazo y prevención al establecer nuevos vínculos sociales con personas que comparten el diagnóstico, la negación a exponerse al fracaso, juzgamiento y censura por los demás participantes de los talleres. Las anteriores razones limitan la sensación de satisfacción y gratificación que 
se pueden experimentar en los procesos realizantes y en el desarrollo de sus cualidades humanas, pero que representaba seguridad ante lo ya conocido.

La dinámica grupal permitió favorecer los propósitos de socialización entre los participantes en cuanto se posibilitó la toma de decisiones conjunta dando soluciones efectivas y pertinentes a las situaciones planteadas en las actividades. Asi mismo se percibieron actitudes de solidaridad, compañerismo y apoyo intragrupal posibilitaba gratificación e interacción satisfactoria.

El desarrollo de las actividades permitió un crecimiento recíproco en la dinámica interna (terapeutas-usuarios), y la retroalimentación de experiencias. Este factor fue favorecido por la orientación humanista e integradora de lo individual con las colectividades dado por la aproximación teórica realizada.

\subsubsection{Atención Indirecta}

Este tipo de atención dirigida a la pareja, familiar o amig@ de la persona asistente al programa VIHDA, tuvo como finalidad promover el codesarrollo de metas terapéuticas y potencializar el desempeño ocupacional dentro del escenario social primario. Aunque se hizo citación vía telefónica, en cartelera y personal a los asistentes a los talleres y formulaciones, no se obtuvo respuesta de las personas convocadas a este senvicio. Esto evidencia que el impacto a nivel del núcleo social de la persona que vive con Vih/Sida es también significativo y que refleja la negación de la condición de salud de su familiar, el anonimato, el reproche o el desconocimiento de ésta.

Por tanto, el espacio destinado para el trabajo indirecto, los participantes de VIHDA identificaron y proyectaron la utilidad y necesidad de la articulación de redes de apoyo a nivel familiar-social $\mathrm{e}$ institucional, identificando grupos de autoapoyo, programas y servicios estatales y no estatales, entre otros. Al realizar esta actividad se evidenciaron vínculos disfuncionales e inexistentes, que en algunos casos obedecían al temor al estigma social, al aislamiento y pocos puntos de apoyo en las redes realizadas, ante lo cual las terapeutas brin-
Las estrategias utilizadas para el desarvollo de estas actividades estuvieron orientadas a que en la buisqueda del sentido gratificante en el desempeño ocupacional se incluya como determinantes el (ve) conocimicnto personal, la autoconciencia corporal, la elaboración de procesos de duelo, la estructurración de bábitos y mitinas saludables, la utilización productiva del tiempo libre y el sentido de vida daron opciones de vinculación a programas que favorezcan el desarrollo de los propósitos realizantes.

\subsubsection{Historias de vida}

A partir de las experiencias de VHIDA originadas por la interacción con las personas asistentes al programa, se presentan en el trabajo original 3 historias de Vida, que plasman la cotidianidad del vivir con Vih/Sida y como las categorías sociales, mencionadas con anterioridad, marcan pautas importantes dentro del desempeño ocupacional. Se describe paralelamente el análisis desde terapia ocupacional en cuanto a la conjunción o compensación entre implicaciones físicas, mentales, sociales y espirituales y sus propósitos tendientes hacia la realización y teniendo en cuenta la relación única e individual establecida con su entorno social y familiar, y en últimas, frente a sí mismas.

Con esta reconstrucción, se pretende, acercar al lector a la realidad multifacético del Vih/Sida y contribuir al cambio positivo en la concepción, principalmente en la actitud entre seres humanos; y quizás descubrir nuevas significancias del virus no solo en cuanto a lo trágico, lo devastador y lo infeccioso, que bastante difusión ha tenido ya en la sociedad, sino también lo positivo, lo espiritual, lo constructivo, el renacer y el reconocer en el vivir o convivir con el VIH/SIDA "Valores e llusiones que Humanizan"

\footnotetext{
ro Parafraseando a Juan Carlos Riascos en entrevista televisada a proposito de su libro "En el laberinto de la esperanza"
}

Revista Ocupación Humana|37 


\section{Conclusiones y Recomendaciones}

El diseño, ejecución y posterior sistematización del Programa VIHDA, permitieron a las autoras identificar diversas variables útiles en el desarrollo de una pasantía dirigida a personas que viven con $\mathrm{VIH} / \mathrm{SIDA}$. Con el presente escrito se espera contribuir al enriquecimiento de la perspectivas de terapia ocupacional frente al VIH/SIDA y promover nuevos planteamientos que apoyen las acciones académicas e institucionales desarrolladas en Colombia.

- Se sugiere a las personas o instituciones interesadas en el abordaje del VIH/SIDA, considerar la importancia de desarrollar o adaptar las estrategias planteadas por el programa VIHDA. Se espera el aporte teórico y práctico que se registra en el presente escrito, sea replicable en cuanto al desarrollo de las estrategias de valoración e intervención; a la utilización del instrumento para la Valoración del Desempeño Ocupacional Realizante; a las contribuciones al modelo Desempeño Ocupacional Realizante y a la propuesta para el abordaje de personas que viven con VIH/SIDA.

- La dificultad e impacto que representa para la sociedad el convivir con el VIH/SIDA y las implicaciones en cuanto al estigma social que se tejen al respecto, fueron puestas en evidencia con las respuestas obtenidas en el proceso de Valoración y con la no asistencia de acudientes a las actividades programadas para la atención indirecta. Se recomienda a quienes trabajan a favor de las personas que viven con $\mathrm{VIH} / \mathrm{SIDA}$, consideren la necesidad de involucrar en el tratamiento a personas que conviven con $\mathrm{VIH} / \mathrm{SIDA}$ como apoyo para el mantenimiento y mejoramiento de la calidad de vida individual y colectivo. Así mismo se sugiere que terapia ocupacional y las demás disciplinas involucradas en la atención de esta población, desarrollen estrategias de intervención transdisciplinaria (en clínica o domicilio) para las personas que se encuentran en fase de exacerbación de síntomas y por tanto tienen limitado el desplazamiento y acceso a este tipo de programas.

- Con referencia al perfil del terapeuta ocupacional, a partir de la experiencia en el programa VIHDA se evidenció la necesidad del trabajo del terapeuta ocupacional. Es preciso complementar estos saberes con un perfil personal que permita al terapeuta actuar como un medio facilitador del desempeño ocupacional realizante. Se sugiere a los terapeutas ocupacionales en formación 
dar continuidad a este tipo de experiencias prácticas, y. a los profesionales valorar y adaptar las estrategià aquí presentes, para la intervención para la valoración e interverición de esta población. Así mismo se recomienda el trabajo Inter y trasdiciplinario para con esta población. Sobre el desarrollo de la investigación de corte cualitativo bajo modalidad pasantía, sê puede recomendar como : de gran utilidad la estrategia investigativa de sistematización para presentar y analizar resultados a nivel conceptual, metodológico y personal, y contribuir al conocimiento más allá de la presentación de un informe final exclusivamente. La sistematización permitió reconstruir, comprender y trasformar las experiencias y conocimientos generados a partir del desarrollo del programa VIHDA, siguiendo una articulación dialéctica de teoría - práctica - teoría. Para las autoras dicha opción investigativa representó un aporte significativo, en cuánto permitió evidenciar al cumplimiento de los objetivos propuestos en el proyecto y dè las expectativas personales $y$ académicas de la pasantía.

\%ONUSIOA. Resumen nundial de la epidemia de VIH SLDA. Revisado en Pag Web wwwonusida.org.com 2003 .

2. Beltran Manuel y cols. V Estudio centinela Nacio nalla de Vigilancia de infeccion por vi 1 colombian informe epidemiologico quincenal Namonal. Volos 5 o

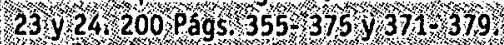

3 Galns angie \& Veloza, Adnana. programa dejerapia ocupacionalgara personas con vî/SIOA Unjversidad Nacionalde colombia. fraculta d de Medicina carrèra de Terapia ocupacional 1999

\% Murphy, Nancy y cols; kentoprogram a nodel of interdisciplinany and inter dependent care en Amencan ou ounal of occupational

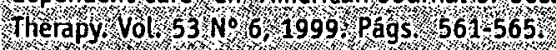

F Caicedo bibianay cols. Intervención de Térapia ocupactonalsobrel los efectos psicosociales antela respuesta en pacientes con viH fundacion Universi tana Maniela Beltran Carrera de Terapia ocupacional 1996

6 Diaz, Magday cols. xerapia ocupacional para per

sonas con enfermedad incurable tuna vision desde la
Logoterapia. Universidad Nacconal de colombia. Facul tad de Medicina carrera de terapia ocupacional 2001

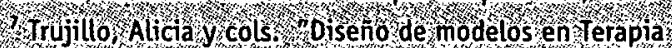
0 cupaciona en Terapia ocupacional y Universidad. Universidad Naciona lo colombia, $1994, \mathrm{pags}, 91: 98$ 8 - Modelo Desempeno ocúpacionaly realización humana. suóngen y alcances en Terapia ocupacional y universdad. Wniversidad Nacionalde colombia. $1994 \%$ pas. 99 , 114

9 Grupo ocupacion y Relizacióntomana oefinición conceptualde los componentes del modelo oesem peño ocupacional Realizante. Documento en cons truccón. Universidad Nacional décolombia. acultad de Medicnna oepartamento de terapias Unidad de ta ocupacion tumana: abri 2001

10 Trujillo Aliciar oesempeno Ocupacional Reali zante: un mo delo sobre los propositos de la Terapla

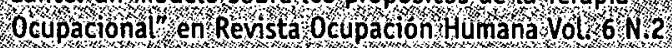
1995. pag 12 .

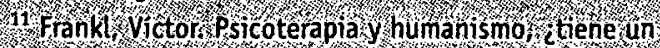

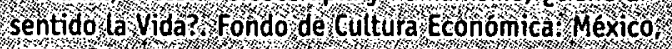
1978. $\mathrm{pag} .37$ 